\title{
A mini-review on asthma
}

\begin{abstract}
Asthma is the most common disease of respiratory system. Even after the advancement of diagnosis, management of this disorder is not under control. In major cases, avoidance measures are used to achieve control. Inhaled corticosteroids (ICSs) are the centre point for the treatment. If ICS therapy is not able to control the situation in adults then combination ICS/long-acting beta2-agonists (LABA) inhalers are used. Allergen-specific immunotherapy represents a potentially disease-modifying therapy for many patients with asthma, but it should be prescribed by only those physicians who are properly trained for allergens. Asthma can be controlled by regular monitoring and adherence to therapy. Inhaler techniques are also one of the essential components of asthma management. This article provides a review of the definition, diagnosis and currently used techniques for the treatment of asthma.
\end{abstract}

Keywords: asthma, inhalers, allergens, ICSs, LABA
Volume 7 Issue I - 2018

\author{
Aditya Dixit, Prashant Kumar Dhakad \\ Department of Pharmacy, School of Medical and Allied Sciences \\ Galgotias University, India
}

Correspondence: Aditya Dixit, Department of Pharmacy, School of Medical and Allied Sciences Galgotias University,

Yamuna Expressway, Gautambudh Nagar, U.P, India,

Email aditya.dixit@galgotiasuniversity.edu.in

Received: January 04, 2018 | Published: January 10, 2018

\section{Introduction}

Asthma has not been satisfacotorily defined ever, even when it is the most common disease of childhood. ${ }^{1}$ It affect almost $10 \%$ of population in Canada that's why it is the most common chronic respiratory disorder. ${ }^{2}$ Though it is often believed to be a disorder limited to lungs only but recent studies indicates that it may represent a component of systemic airway disease involving the whole respiratory system, and this is supported by the fact that asthma and other atopic disorders coexist very frequently, particularly allergic rhinitis. ${ }^{3}$ The symptoms of asthma generally include recurrent wheezing, coughing, chest tightness, and dyspnea, with nightly and early morning symptoms being more prevalent, whereby quality of life is often reduced. ${ }^{4}$ These symptoms may already occur early in life, with approximately one third of child population shows wheezing during the first three years of age. ${ }^{5}$ Majority stops wheezing till six years of age, $40 \%$ will continue to wheeze and they have already developed asthma or developing asthma at a late stage. Dependent on questioning methodology, up to $10-15 \%$ of children may suffer from asthma complaints by school age. ${ }^{6}$ In many children, the severity of symptoms disappears at early puberty and may even diminishes completely, especially in cases of mild asthma. However, it is well understood and accepted that symptoms may remain in children with severe asthma or they can return in adulthood.?

\section{Definition}

Asthma is defined as a chronic disease of the inflammation in respiratory tract. The chronic inflammation is due to hyperresponsiveness of cells of airway (an exaggerated airway narrowing response to triggers, such as allergens and exercise), which leads to recurrent symptoms such as wheezing, dyspnea (shortness of breath), chest tightness and coughing. Symptom episodes are generally associated with widespread, but variable, airflow obstruction within the lungs that is usually reversible either spontaneously or with appropriate asthma treatment. ${ }^{8}$

\section{Diagnosis}

The diagnosis of asthma involves a thorough medical history, physical examination, and objective assessments of lung function (spirometry preferred) to confirm the diagnosis. Bronchoprovocation challenge testing and assessing for markers of airway inflammation also assists for diagnosing the disease, specifically when objective measurements of lung function are normal despite the presence of symptoms of asthma. ${ }^{8-10}$

\section{Treatment}

Avoidance measures: If relevant allergens/irritants are avoided then it is an important component of asthma management. Those patients who are allergic to house dust mites should be instructed to use covers which are allergen impermeable and which keep the relative humidity in the home below $50 \%$ (to inhibit mite growth). Cigarette smoking and exposure to second-hand tobacco smoke should also be avoided. ${ }^{10}$

Reliever medications: Rapid-acting beta2-agonists which can be inhaled are the preferred reliever medications for treatment of acute symptoms, and they should be prescribed to all patients with asthma. In Canada, various Short-acting beta2-agonists (SABAs; e.g., salbutamol, terbutaline) and one LABA (formoterol) are approved for this indication. ${ }^{8-10}$ Furthermore, chronic, short-acting anticholinergic bronchodilator therapy is not recommended for use in children. ${ }^{9}$

\section{Controller medications}

Inhaled corticosteroids (ICSs): ICSs are the most effective antiinflammatory medications which are available for the treatment of asthma and they represent the centre point of therapy for most patients. Low-dose ICS monotherapy is recommended as first-line maintenance therapy for most children and adults with asthma. ${ }^{8-10}$

Leukotriene receptor antagonists (LTRAs): The LTRAs montelukast and zafirlukast are also effective for treatment and they are generally considered to be safe and they are also well tolerated. However, ICS treatment is more effective than these agents, hence; they are generally reserved for patients who are not able to use ICSs. LTRAs can also be used as supplement therapy if asthma is uncontrolled despite the use of low-to-moderate dose ICS therapy. It is important to note, however, that LTRAs are considered to be less effective than LABAs as add-on therapy in adults. ${ }^{8,9}$

Theophylline: Theophylline is an oral bronchodilator with some anti-inflammatory effects. Due to its narrow therapeutic window and frequent adverse events (e.g., gastrointestinal symptoms, loose stools, seizures, cardiac arrhythmias, nausea and vomiting), it is only used for patients who have uncontrolled asthma despite an adequate trial of ICS, LABAs and/or LTRAs. ${ }^{8,9}$ 
Allergen-specific immunotherapy: In this, gradually increasing quantities of the patient's relevant allergens are administered subcutaneously until a dose is reached that is effective in inducing immunologic tolerance to the allergen. It has been widely used to treat allergic asthma even then it is not universally accepted by all clinical practice guideline committees due to the potential for serious anaphylactic reactions with this form of therapy. ${ }^{11}$ A Cochrane review of 75 randomized controlled trials examining the use of allergenspecific immunotherapy in asthma management confirmed its efficacy in reducing asthma symptom scores and medication requirements, and improving airway hyper responsiveness. ${ }^{12}$ Sublingual immunotherapy has shown similar benefits and has been noted with, ${ }^{13}$ which is expected to be approved in Canada in the near future. Evidence also support that allergen-specific immunotherapy can prevent the onset of asthma in atopic individuals. ${ }^{14}$

\section{Discussion}

a. A clinical diagnosis of asthma should be suspected in patients with intermittent symptoms of wheezing, coughing, chest tightness and breathlessness.

b. Objective measurements of lung function, preferably using spirometry, are needed to confirm the diagnosis.

c. A rapid-acting bronchodilator's use should be prescribed to provide relief of acute symptoms for all asthma patients.

d. ICS therapy is the standard of care for most patients with asthma.

e. Those adult patients who fail to achieve control with low-tomoderate ICS doses are recommended to use combination ICS/ LABA inhalers.

f. If asthma is uncontrolled even after the use of low-to-moderate ICS doses, LTRAs can also be used as supplement therapy.

g. Allergen-specific immunotherapy is very potential diseasemodifying therapy that can be considered in various cases of allergy associated asthma.

h. Asthma control should be regularly monitored, patient should adhere to therapy and asthma should be managed with inhaler techniques.

\section{Acknowledgments}

All the facilities were provided by the Galgotias University, I am really thankful to all the staff members for supporting my research work.

\section{Conflicts of interest}

None of the author has any conflict of interest in the context of this work.

\section{References}

1. Anderson HR, Bailey PA, Cooper JS, et al. Morbidity and school absence caused by asthma and wheezing illness. Arch Dis Child. 1983;58(10):777-784.

2. http://www.phac-aspc.gc.ca/

3. Bourdin A, Gras D, Vachier I, et al. Upper airway 1: Allergic rhinitis and asthma:united disease through epithelial cells. Thorax. 2009;64:9991004.

4. Van Aalderen WMC, Meijer GG, Oosterhoff Y, et al. Epidemiology and the concept of underlying mechanisms of nocturnal asthma. Resp Med. 1993;87:37-39.

5. Martinez FD, Wright AL, Taussig LM. Asthma and wheezing in the first six years of life. N Eng J Med. 1995;332(3):133-138.

6. Mommers M, Gielkens-Sijstermans C, Swaen GMH, et al. Trends in the prevalence of respiratory symptoms and treatment in Dutch children over a 12 year period:results of the fourth consecutive survey. Thorax. 2005;60(2):97-99.

7. Gerritsen J. Follow-up studies of asthma from childhood to adulthood Paed Resp Rev. 2002;3(3):184-192.

8. http://www.ginasthma.com

9. Lougheed MD, Lemière C, Dell SD, et.al. Canadian Thoracic Society asthma management continuum:2010 consensus summary for children six years of age and over, and adults. Can Respir J. 2010;17(1):15-24.

10. Kaplan AG, Balter MS, Bell AD, et al. Diagnosis of asthma in adults. CMAJ. 2009;181(10):E210-E220.

11. Frew AJ. Allergen immunotherapy. $J$ Allergy Clin Immunol. 2010;125:S306-S313.

12. Abramson MJ, Puy RM, Weiner JM. Allergen immunotherapy for asthma. Cochrane Database Syst Rev. 2003;2:CD001186.

13. Calamita Z, Saconato H, Pela AB, et al. Efficacy of sublingual immunotherapy in asthma:systematic review of randomized clinical trials using the Cochrane Collaboration method. Allergy. 2006;61(10):11621172 .

14. Grembiale RD, Camporota L, Naty S, et al. Effects of specific immunotherapy in allergic rhinitic individuals with bronchial hyperresponsiveness. Am J Respir Crit Care Med. 2000;162(6):20482052 . 